\title{
In vivo treatment with M8, a highly diluted tinctures complex, reduced the malignancy of a mouse melanoma model.
}

\author{
Lucas Ferrari de Andrade, Fernando de Souza Fonseca Guimarães, \\ Gustavo Rossi, Rafael Zotz, Eneida J. Da Lozzo, \\ Célia Regina Cavichiolo Franco, Carolina Camargo de Oliveira, \\ Dorly de Freitas Buchi, Edvaldo da Silva Trindade
}

Univesidade Federal do Paraná, Curitiba, PR, Brasil.

\begin{abstract}
Background: Cancer is a class of disease responsible for $13 \%$ of death cause worldwide. Among all types of cancers, one of the most aggressive and with the highest death rate is melanoma. It is highly metastatic and current treatments with chemotherapeutic drugs do not yield satisfactory results. Therefore, the interest on new therapeutics for cancer treatment has been increasing on research. Highly diluted tinctures (HDT) are intended to enhance immune system responses resulting in reduced frequency of various diseases, and often present no risk of serious side-effects due to its low toxicity. Previous results have demonstrated in vitro inhibition of invasion ability and in vivo anti-metastatic potential of B16F10 lung metastasis model after mice treatment with M8 inhalation.
\end{abstract}

Aims: Now we have evaluated M8 effects on hyaluronic acid and its specific melanoma cell surface receptor (CD44) expression on lungs after inhalation by mice.

Methodology: M8 compounds include Aconitum napellus 20dH, Arsenicum album 18dH, Asa foetida 20dH, Calcarea carbonica 16dH, Conium maculatum 17dH, Ipecacuanha 13dH, Phosphorus 20dH, Rhus toxicodendron 17H, Silicea 20dH, Sulphur 24dH, and Thuja occidentalis 19dH. B16F10 Melanoma cells were inoculated into C57B/L6 mouse lateral tail vein. Treatment started 24 hours after inoculation, and was repeated after each 12 hours during 14 days on an inhalation chamber that is adapted to little rodents. Mice were subjected to euthanasia by intraperitoneal injection of thiopental followed by decapitation. Lungs were surgically removed and analyzed under a stereomicroscope for the presence of metastatic foci. They were formaldehyde fixed, dehydrated and paraffin embedded. Histological sections were processed for hematoxilin/eosin (HE), Fontana-Masson and immunohistochemistry staining methods. Images were captured and blindly analysed by ImageJ (NIH) software.

Results: HE and Fontana-Masson showed a reduction in number and size of metastatic nodules, as previously demonstrated. We have detected a reduction on hyaluronic acid as well as CD44 expression on mice lungs after M8 treatment. The high metastatic potential of melanoma is proportional to hyaluronic acid expression level, together with its specific cell surface receptor, the CD44. These results suggest that M8 treatment reduces malignancy of mouse melanoma through modulation of hyaluronic acid and CD44 expression, which play crucial roles in tumor invasion and growth. 
Conclusion: Even though further investigation are necessary to elucidate the mechanisms of action of M8 treatment there is an indication that these highly diluted tinctures could be a promising therapy to treat metastatic melanoma.

Keywords: High diluted tinctures, mouse melanoma, Hyaluronic Acid, CD44.

\section{Tratamento in vivo com M8, uma solução complexa altamente diluída, reduziu a malignidade de um modelo de melanoma de camundongo.}

\section{RESUMO}

Introdução: O Câncer é uma classe de doenças responsáveis por 13\% das causas de mortes no mundo todo. Entre todos os tipos de cânceres, um dos mais agressivos e com maior índice de mortalidade é o melanoma. Ele é altamente metastático, e os tratamentos atuais com drogas quimioterapeuticas não geram resultados satisfatórios. Portanto, o interesse em novos agentes terapeuticos para o tratamento do câncer tem aumentado na pesquisa. Soluções altamente diluídas $(\mathrm{CAD})$ são destinadas à aumentar a resposta do sistema imunológico resultando em menores frequencias de várias doenças, e também não apresentam riscos de graves efeitos colaterais, devido à sua toxicidade reduzida. Resultados anteriores demostraram a inibição in vitro da habilidade de invasão e potencial antimetastático in vivo do modelo de metástase pulmonar da B16F10, após o tratamento dos camundongos pela inalação do M8.

Objetivos: Nós agora avaliamos os efeitos do M8 na expressão de ácido hialurônico e seu receptor (CD44) na superfície das células de melanoma em pulmões de camundongos após inalação.

Metodologia: O composto M8 é constituído de Aconitum napellus 20dH, Arsenicum album 18dH, Asa foetida $20 \mathrm{dH}$, Calcarea carbonica 16dH, Conium maculatum 17dH, Ipecacuanha 13dH, Phosphorus 20dH, Rhus toxicodendron $17 \mathrm{H}$, Silicea $20 \mathrm{dH}$, Sulphur 24dH, e Thuja occidentalis $19 \mathrm{dH}$. Células de melanoma B16F10 foram inoculadas na veia lateral de camundongos C57B/L6. O tratamento foi iniciado 24 horas após a inoculação, e foi repetido após cada 12 horas, até completar 14 dias, em uma câmara de inalação adaptada para pequenos roedores. Posteriormente, camundongos foram sujeitados à eutanásia atravéz de injeção intraperitoneal de tiopental seguida por decaptação. Os pulmões foram cirurgicamente removidos e analizados em um estereomicroscópio para a presença de nódulos metastáticos. Em seguida, eles foram fixados em fomaldeído, desidratados e emblocados em parafina. Cortes histológicos foram processados para os métodos de coloração hematoxilina/eosina (HE), Fontana-Masson e imunohistoquímica. As imagens foram capturadas e analisadas de maneira cego pelo software ImageJ (NIH).

Resultados: As colorações de HE e Fontana-Masson mostraram uma redução no número e tamanho dos nódulos metastáticos, como previamente demonstrado. Nós detectamos uma redução nas espressões de ácido hialurônico assim como de CD44, no pulmão dos camundongos após tratamento com M8. O alto potencial metastático do melanoma é proporcional ao nível de expressão de ácido hialurônico, conjuntamente com seu receptor específico de superfície celular, o CD44. Esses resultados sugerem que o tratamento com M8 reduz a malignidade do melanoma de camundongo através da modulação das expressões de ácido hialurônico e CD44, os quais desempenham funções cruciais na invasão e crescimento tumoral. 
Conclusão: Mais pesquisas são necessárias para esclarecer os mecanismos de ação do tratamento com M8. Entretanto, há um indicativo de que soluções altamente diluídas podem ser terapias coadjuvantes para o tratamento do melanoma metastático.

Palavras-chave: Soluções altamente diluídas, melanoma de camundongo, ácido hialurônico, CD44.

\section{(c)) BY-NC-ND Licensed to GIRI}

Support: We thank CAPES, CNPq and SETI for financial support. We had full access to all the data in this study and we take complete responsibility for the integrity of the data and the accuracy of the data analysis.

Conflict of interest: authors declare there is no conflict of interest

Correspondence author: Dorly de Freitas Buchi, labbiocel@ufpr.br

How to cite this article: Andrade LF, Guimarães FSF, Rossi G, Zotz R, Lozzo EJD, Franco CRC, de Oliveira CC, Buchi DF, Trindade ES. In vivo treatment with M8, a highly diluted tinctures complex, reduced the malignancy of a mouse melanoma model. Int J High Dilution Res [online]. 2011 [cited YYYY Month dd]; 10(36): 142-144. Proceedings of the XXV GIRI Symposium and VIII CBFH; 2011 Sep 04-07; Foz do Iguaçu (Brazil). GIRI and ABFH; 2011; Available from: http://www.feg.unesp.br/ ojs/index.php/ijhdr/article/view/481/492 